\title{
Guidelines for Quality Control Testing of Molecular Breast Imaging Systems
}

\author{
Sara M. Nardinger, CNMT, Thuy D. Tran, CNMT, Tiffinee N. Swanson, CNMT, Lacey R. Ellingson, CNMT, \\ Courtney M. Solberg, CNMT, Michael K. O’Connor, PhD, and Carrie B. Hruska, PhD \\ Department of Radiology, Mayo Clinic, Rochester, Minnesota
}

\begin{abstract}
Molecular breast imaging $(\mathrm{MBI})$ is a nuclear medicine test that uses dedicated $y$-cameras designed for imaging of the breast. Despite growing adoption of $\mathrm{MBI}$, there is currently a lack of guidance on appropriate quality control procedures for MBI systems. Tests designed for conventional $y$-cameras either do not apply or must be modified for dedicated detectors. Our objective was to provide practical guidance for physics testing of $\mathrm{MBI}$ systems by adapting existing quality control procedures for conventional systems. Methods: Quality control tests designed for conventional $\mathrm{y}$-cameras were attempted on a dedicated $\mathrm{MBI}$ system and then modified as necessary to accommodate the pixelated detector, limited space between dual-detector heads, and inability to fully rotate the detector gantry. Results: $\mathrm{MBI}$ systems were found to warrant quality control testing of uniformity, spatial resolution, count sensitivity, energy resolution, and lesion contrast. The modified procedures and special considerations needed for these tests were investigated and described. Physics tests of intrinsic uniformity, count rate parameters, and overall system performance for SPECT did not apply to dedicated $\mathrm{MBI}$ systems. Conclusion: Routine physics testing of dedicated MBI equipment is important for verifying system specifications and monitoring changes in performance. As adoption of $\mathrm{MBI}$ grows, routine testing may be required for obtaining and maintaining accreditation from regulatory bodies.
\end{abstract}

Key Words: molecular breast imaging; quality control; dedicated gamma camera

J Nucl Med Technol 2018; 46:349-354

DOI: 10.2967/jnmt.118.209221

$\mathbf{T}$ he goal of physics testing of clinical imaging equipment is to ensure optimal performance and accurate imaging. Routine testing is important for monitoring changes in system performance and may be needed for obtaining and maintaining accreditation from regulatory bodies. However, there is currently a lack of resources available to guide quality control procedures specific to dedicated $\gamma$-cameras used for molecular breast imaging (MBI) procedures.

Received Feb. 15, 2018; revision accepted Apr. 24, 2018.

For correspondence or reprints contact: Sara M. Nardinger, Department of

Radiology, Charlton 1, Mayo Clinic, 200 First St. S.W., Rochester, MN 55905.

E-mail: nardinger.sara@mayo.edu

Published online Jun. 8, 2018.

COPYRIGHT (c) 2018 by the Society of Nuclear Medicine and Molecular Imaging.
Several types of dedicated MBI cameras are currently available (1). Here, we refer to MBI as imaging systems comprising detectors designed for single-photon-emitting radionuclides and not dedicated breast PET imaging systems. Some dedicated MBI detectors comprise pixelated arrays of sodium iodide coupled to position-sensitive photomultiplier tubes (also known as breast-specific $\gamma$-imaging (2)). Other detector configurations include a multicrystal array of cesium iodide coupled to solid-state silicon photodiodes (3) and a completely solid-state detector that uses cadmium-zinc-telluride (2). Collimators for MBI systems can vary in design. Some have a standard hexagonal-hole collimator, whereas others have a square-hole collimator matched to the individual square detector elements (4). MBI units can also have single or dual detector heads. In all these systems, the size of the detector is reduced relative to that of a conventional $\gamma$-camera, to accommodate positioning of the breast directly on or close to the detector. Differences between dedicated MBI and conventional $\gamma$-cameras, such as the smaller detector size, pixelated detectors, and the inability to accommodate standardsized phantoms, must be considered when performing physics testing because many of the conventional tests either do not apply or must be modified for dedicated systems.

Here, we provide practical guidance for physics testing of MBI systems by adapting existing quality control procedures for conventional systems.

\section{MATERIALS AND METHODS}

Quality control tests designed for conventional $\gamma$-cameras were attempted on a dedicated MBI system and then modified as necessary to accommodate the pixelated detector, limited space between dual-detector heads, and inability to fully rotate the detector gantry.

\section{RESULTS}

Table 1 summarizes the recommended quality control procedures and testing frequencies for MBI systems.

\section{Uniformity Testing}

Uniformity testing of the MBI detectors needs to be performed each day of operation, similarly to the requirement for conventional nuclear medicine $\gamma$-cameras. Integral uniformity should be evaluated. Other uniformity metrics 
TABLE 1

Recommended Quality Control Testing Program for MBI Systems

\begin{tabular}{|c|c|c|c|c|}
\hline Test & Equipment & Frequency & Acquisition details & Passing criterion \\
\hline Uniformity & $\begin{array}{l}{ }^{57} \text { Co sheet source } \\
\text { or fillable phantom }\end{array}$ & Daily & 7.5 million counts & $\leq 5 \%$ integral uniformity \\
\hline Spatial resolution & 4-quadrant bar phantom & Semiannually & $\begin{array}{l}7.5 \text { million counts; phantom } \\
\text { angled across field of view }\end{array}$ & $\begin{array}{l}\text { Meets manufacturer's } \\
\text { specifications }\end{array}$ \\
\hline Sensitivity & Flask & Annually & 120 -s images & $\begin{array}{l}\leq 10 \% \text { difference between } \\
2 \text { detectors }\end{array}$ \\
\hline Energy resolution & Point source & Annually & $\begin{array}{l}\text { 2-keV energy windows; } 1 \text {-min } \\
\text { images }\end{array}$ & $\begin{array}{l}\text { Full width at half maximum } \\
\quad \leq 10 \%\end{array}$ \\
\hline Lesion contrast & Contrast-detail phantom & Quarterly & $\begin{array}{l}1 \text { million counts; images at } \\
3 \text { depths }\end{array}$ & $\begin{array}{l}\text { CNR }>3 \text {; count number of } \\
\text { visible lesions at each depth }\end{array}$ \\
\hline
\end{tabular}

*All tests should be performed at acceptance testing and after major service work.

$\mathrm{CNR}=$ contrast-to-noise ratio.

used by some manufacturer software programs, such as SD divided by the mean, are not sufficient for measuring uniformity of the detectors because these calculations are not consistent with National Electrical Manufacturers Association standards (5). Some MBI units have a fixed collimator that is not designed to be removed. For these systems, only extrinsic uniformity should be evaluated because removing the collimator may compromise the system.

To acquire the uniformity flood image, either a sheet source or a refillable flood source can be used. A refillable flood source with ${ }^{99 \mathrm{~m}} \mathrm{Tc}$-pertechnetate $\left(\mathrm{TcO}_{4}{ }^{-}\right)$is fast and easy to use and is less expensive than purchasing ${ }^{57} \mathrm{Co}$ sheet sources. If a refillable source is used, it should be slightly larger than the detector field of view to ensure that the entire detector is covered by the source. This smallersized flood source greatly facilitates handling and adequate mixing of the $\mathrm{TcO}_{4}{ }^{-}$. Although ${ }^{99 \mathrm{~m}} \mathrm{Tc}$-sestamibi is typically the radiopharmaceutical of choice for clinical MBI studies, ${ }^{99}$ Tc-sestamibi should not be used in any acrylic or plastic sources, because it can adhere to the plastic (Fig. 1) and, if used in a flood source, may result in a nonuniform flood.

To acquire uniformity flood images, the source should be sandwiched between the 2 detectors, or between the detector and the compression paddle for single-head systems, and the camera rotated to an angle to move any air bubbles out of the field of view (Fig. 2). Keeping an air bubble in the phantom is helpful for mixing. The same energy window used for clinical imaging should be used for performing physics testing. On cadmium-zinc-telluride-based systems, some imaging centers use a wider energy window such as $110-154 \mathrm{keV}$ instead of the standard $140 \% \pm 10 \% \mathrm{keV}$ $(6,7)$. Acquiring at least 7.5 million counts per detector is sufficient for flood images.

If a ${ }^{57} \mathrm{Co}$ sheet source is used, some additional points should be considered. New ${ }^{57} \mathrm{Co}$ sheet sources often contain high-energy contaminants $\left({ }^{56} \mathrm{Co}\right.$ and $\left.{ }^{58} \mathrm{Co}\right)(8)$. If these contaminants are present, use of a ${ }^{57} \mathrm{Co}$ sheet source for a uniformity correction map can produce artifacts in the 99m Tc imaging studies (Fig. 3). These contaminants have shorter half-lives than ${ }^{57} \mathrm{Co}$ and typically decay away within 6-8 mo. On a conventional $\gamma$-camera, increasing the distance between the source and the detector by placing the source on cups or other smaller objects can help minimize the effects of these high-energy contaminants. However, this approach is not feasible with dedicated systems because there may be insufficient space for placement of cups outside the detector field of view. Also, the MBI collimators, being designed for higher sensitivity, are much more susceptible to high-energy contaminants. Thus, it is important to check for contaminants in new ${ }^{57} \mathrm{Co}$ sheet sources by examining the energy spectra of the camera and allowing any contaminants to decay before allowing routine use of the ${ }^{57} \mathrm{Co}$ source as a surrogate for $\mathrm{TcO}_{4}{ }^{-}$.

On completion of the uniformity flood acquisition, the images should be transferred to an analysis workstation that can perform a uniformity calculation using the $\mathrm{Na}$ tional Electrical Manufacturers Association standard (5).
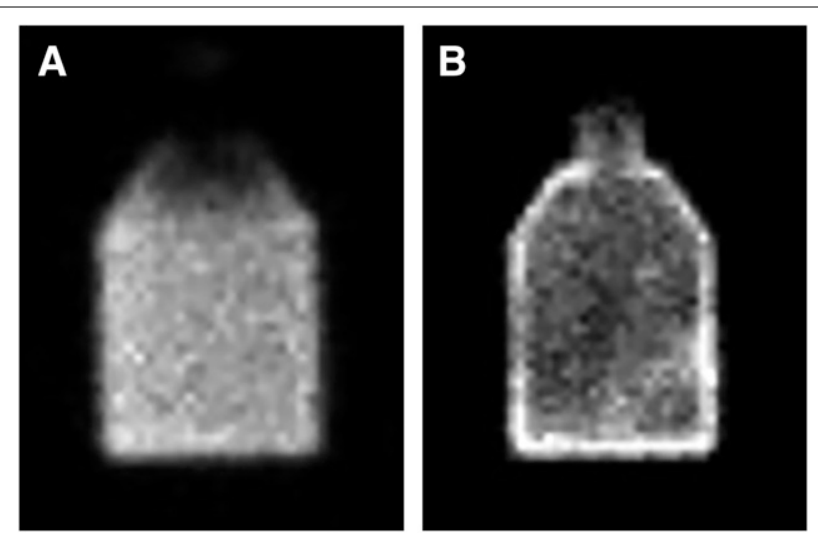

FIGURE 1. Examples of 99mTc-pertechnetate and 99mTcsestamibi in plastic flask. (A) ${ }^{99 m} \mathrm{Tc}$-pertechnetate mixture is homogeneous within flask. (B) Distribution of ${ }^{99 m}$ Tc-sestamibi mixture is nonuniform, and there are increased counts along surface of flask. 


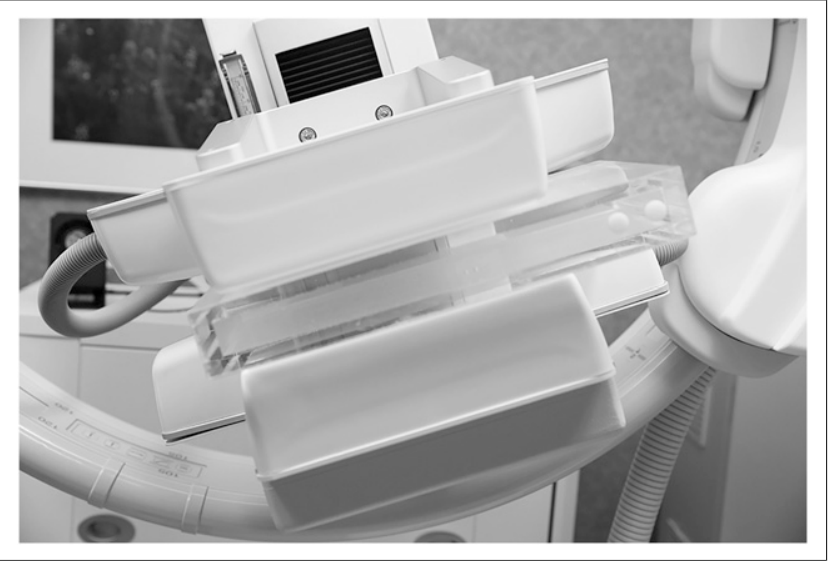

FIGURE 2. Proper positioning for uniformity flood acquisition using refillable $99 \mathrm{mTc}$ source. Source is positioned between upper and lower detectors of dual-head cadmium-zinc-telluride system. Gantry is angled to ensure that any air bubbles in phantom are outside field of view.

The results should show less than 5\% integral uniformity across the field of view.

If individual detector modules are evident or if hot or cold pixels are observed on the daily uniformity flood image, a new uniformity calibration map will need to be obtained according to the manufacturer's directions. Afterward, a uniformity flood image should be acquired again to confirm that the system uniformity is acceptable.

\section{Spatial Resolution Testing}

Unlike a conventional $\gamma$-camera system, the pixelated MBI system has a fixed imaging matrix, with each pixel in the matrix matched to an individual detector element. Because of this design, the resolution of these types of systems does not change over time. Nevertheless, system

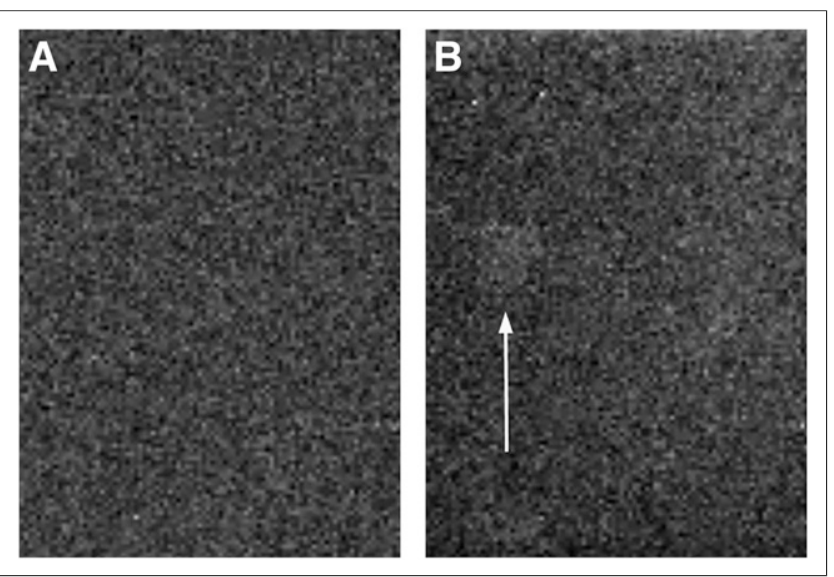

FIGURE 3. Potential of new ${ }^{57} \mathrm{Co}$ sheet sources to create artifacts. (A) Uniformity flood acquisition obtained using ${ }^{57} \mathrm{Co}$ sheet source and ${ }^{57} \mathrm{Co}$ correction map. (B) Uniformity flood acquisition obtained using ${ }^{99 \mathrm{mT}} \mathrm{Tc}$ flood source and ${ }^{57} \mathrm{Co}$ correction map immediately after acquisition of image in A. Artifact (arrow) can be seen over one module in middle of detector. spatial resolution can be evaluated by acquiring a planar image of either a SPECT phantom or a 4-quadrant bar phantom. According to the American College of Radiology, it is recommended that this test be performed at least semiannually on pixelated detectors (9).

Because a standard SPECT imaging phantom does not fit between the detectors of most MBI systems, this test will usually be performed with a bar phantom. Bar phantoms for conventional $\gamma$-cameras may be cumbersome to use as they are quite large (typically $\sim 56 \times 43 \mathrm{~cm}$ ). A bar phantom designed for a cardiac system may be a better option for the smaller detectors of an MBI unit. These phantoms are typically $39 \times 23 \mathrm{~cm}(10)$. Although these phantoms are still larger than the $20 \times 16 \mathrm{~cm}$ detectors, they will be easier to work with and better fit the MBI detectors.

To acquire an image that tests the system resolution, the bar phantom should be placed directly on the lower detector and either a ${ }^{57} \mathrm{Co}$ sheet source or a $\mathrm{TcO}_{4}{ }^{-}$-filled phantom should be placed above the phantom. The detectors should be moved as closely together as possible, tightly sandwiching the source and phantom between the 2 detectors or between the detector and the compression paddle. Rotating the gantry when using a fillable phantom will be useful in moving any air bubble out of the field of view. An acquisition of 7.5 million counts should be sufficient. If using ${ }^{99 \mathrm{~m}} \mathrm{Tc}$, the same energy window should be applied as for clinical imaging. If the system has an upper detector, a second image should be acquired with the radioactive source placed directly on the lower detector and the resolution phantom placed above the source. Again, the detectors should be moved as closely together as possible, tightly sandwiching the source and phantom between the 2 detectors so that the phantom will not move with rotation of the gantry, and 7.5 million counts should be acquired on the upper detector. It is possible that aliasing artifacts may appear with a small bar phantom (Fig. 4A). Angling the phantom across the field of view may help reduce these artifacts.

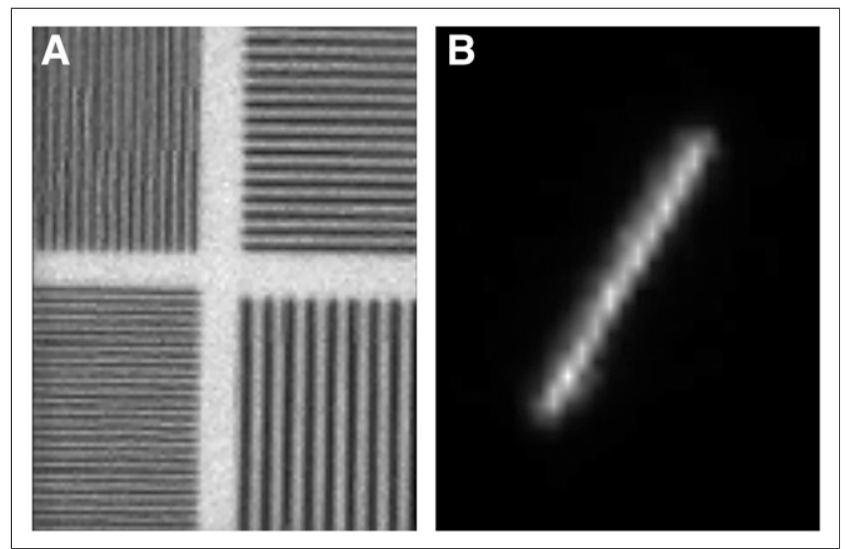

FIGURE 4. Examples of aliasing artifacts on pixelated imaging system. (A) Aliasing artifact from imaging bar phantom. (B) Zoomed image of artifact from line source for evaluating spatial resolution. 
Quantitative analysis of the images can be performed if software is available, or a simple visual analysis can be done by noting the smallest resolvable bar width of the phantom.

Alternatively, the resolution of the MBI system can be tested with a point or line source (Fig. 4B). However, these methods are difficult to perform correctly and can produce inconsistent results (3). Resolution can change with the angle of the line or the position of the point source on the collimator. For these reasons, methods using a line or point source are not recommended for routine spatial resolution testing.

\section{Sensitivity Testing}

The standard criterion for evaluating the relative sensitivity of nuclear medicine $\gamma$-detectors is a difference of less than $5 \%$ between the 2 detectors of a dual-head camera (9). Sensitivity is reported as the number of counts per minute per unit of radioactivity. The standard criterion of less than $5 \%$ may be difficult to achieve with some commercial MBI systems, particularly those with tungsten collimators, for which the manufacturing tolerances are not as tight as for lead collimators.

With a conventional $\gamma$-camera, a flask would be filled with a small volume of water-just enough to cover the surface area of the flask when it is lying on its side (Fig. 5A). Approximately $7.4 \mathrm{MBq}(200 \mu \mathrm{Ci})$ of $\mathrm{TcO}_{4}{ }^{-}$are added to the background volume and mixed thoroughly. The flask is then placed directly on the surface of the collimator, and an image is acquired for $2 \mathrm{~min}$. This procedure is repeated for the second detector when applicable. To calculate the sensitivity, the exact activity of the syringe before and after filling of the flask should be documented, as well as the imaging start time. A sensitivity difference between detector heads of no more than 5\% is considered acceptable. The sensitivity should be within $\pm 10 \%$ of the manufacturer specification, at a defined energy acceptance window.

If the MBI system has only one detector, instead of comparing the sensitivity of one detector with the other, the number of counts per minute is compared with the manufacturer specification.

If the detectors of a dual-detector MBI system are not able to rotate $180^{\circ}$, there are special considerations because the flask cannot be placed directly on each detector. In this case, sensitivity images can be acquired simultaneously with the flask sandwiched between the 2 detectors. The flask should be filled completely with water to minimize differences between detector geometry, the detectors should be rotated slightly to move any air bubbles away from the center of the flask image (Fig. 5B), and a 2-min image should be acquired on both detectors simultaneously. A relative sensitivity difference of less than $10 \%$ is more reasonable to achieve with this type of system.

\section{Energy Resolution Testing}

The energy resolution of an MBI system should be analyzed annually to verify that the scatter rejection is sufficient to provide optimal contrast in clinical studies (9). For MBI systems with collimators that cannot be removed, this test can be performed extrinsically. For most MBI systems, the energy spectrum shown on the acquisition screen does not have enough detail to allow measurement of energy resolution. There are 2 other methods that can be used to calculate the energy resolution: analysis of count data from images acquired at multiple windows distributed across the photopeak, and analysis of count rate.

To calculate the energy resolution using multiple windows across the photopeak, $2-\mathrm{keV}$ energy windows may need to be manually created, depending on the MBI system in use. If a ${ }^{57} \mathrm{Co}$ sheet source is being used, a total of 9 energy windows should be created, each $2 \mathrm{keV}$ wide and ranging from 112 to $130 \mathrm{keV}$. A point source with approximately $5.55 \mathrm{MBq}(150 \mu \mathrm{Ci})$ of ${ }^{99 \mathrm{~m}} \mathrm{Tc}$ may also be used. This source should ideally be centered between the 2 detectors or placed a few centimeters above the lower detector in a single-head system. When ${ }^{99 \mathrm{~m}} \mathrm{Tc}$ is being used, energy windows should be $2 \mathrm{keV}$ wide and range from 130 to $146 \mathrm{keV}$.

An image for each energy window should be acquired for $60 \mathrm{~s}$. The counts acquired in each image can be plotted, or the counts can be analyzed to determine the full width at half maximum and the energy resolution. The results
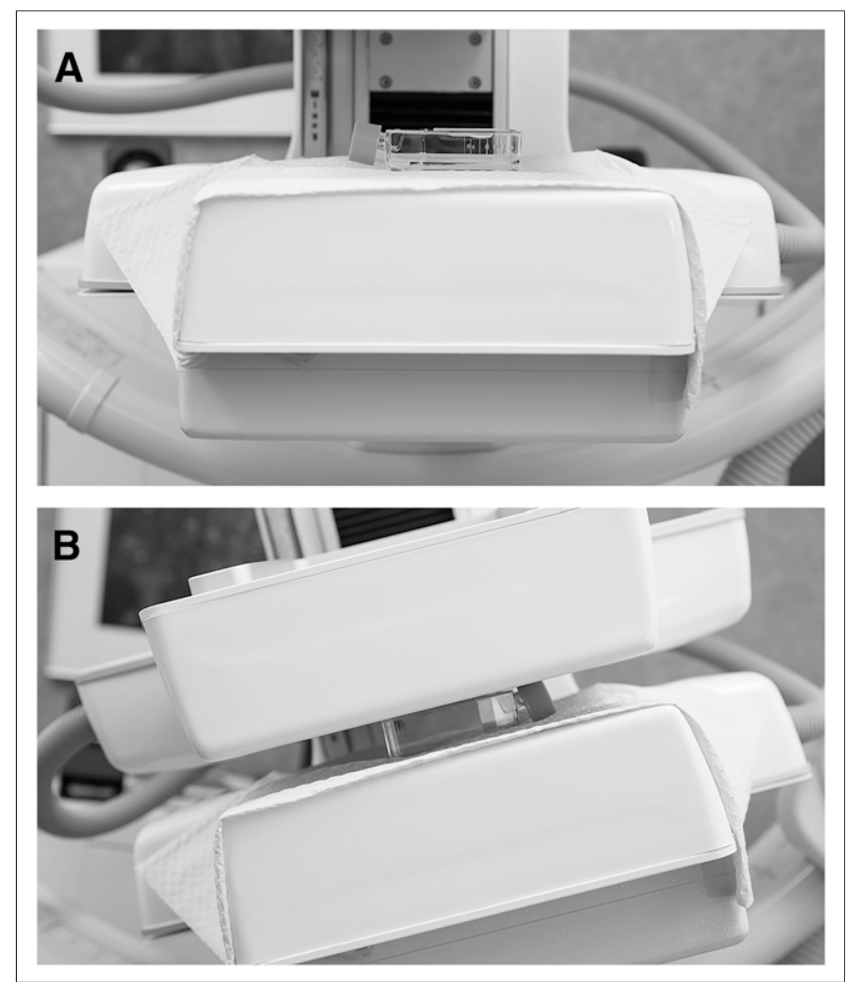

FIGURE 5. Recommended setup for measurement of system sensitivity. (A) For single-head system, flask is filled with just enough water to cover surface area of flask when lying on its side. (B) For dual-head system, flask is filled completely with water to minimize differences between detector geometry. Both images can be acquired simultaneously. 

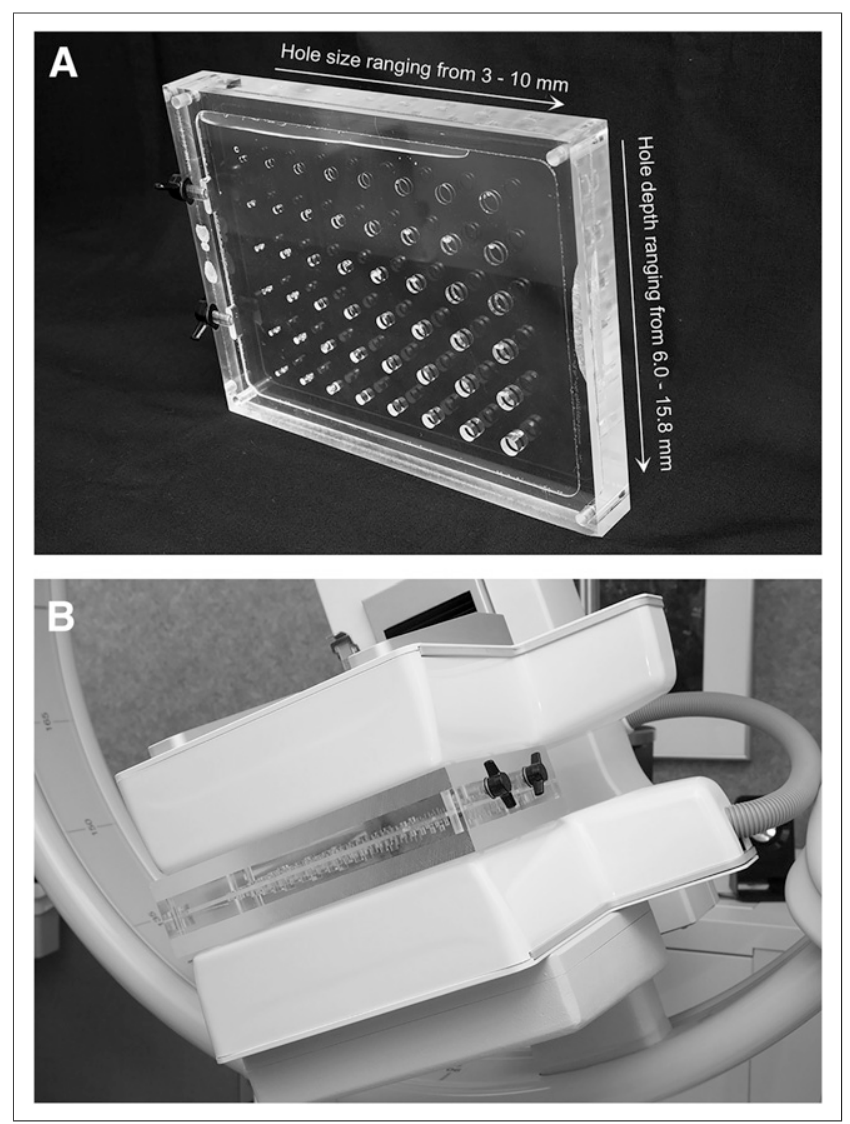

FIGURE 6. Contrast-detail phantom. (A) Phantom contains 3$\mathrm{cm}$-thick central section with multiple hole sizes and depths. (B) Display of image setup using two 1.5-cm-thick acrylic plates on either side of contrast-detail phantom. Camera is angled to move air bubbles out of field of view.

should be compared with the manufacturer specification. This method assesses only part of each detector field of view and may not accurately represent the overall energy resolution.

To estimate the energy resolution using analysis of the count rate, a ${ }^{57} \mathrm{Co}$ sheet source or ${ }^{99 \mathrm{~m}} \mathrm{Tc}$ flood source is placed between the detectors as described above, the peak

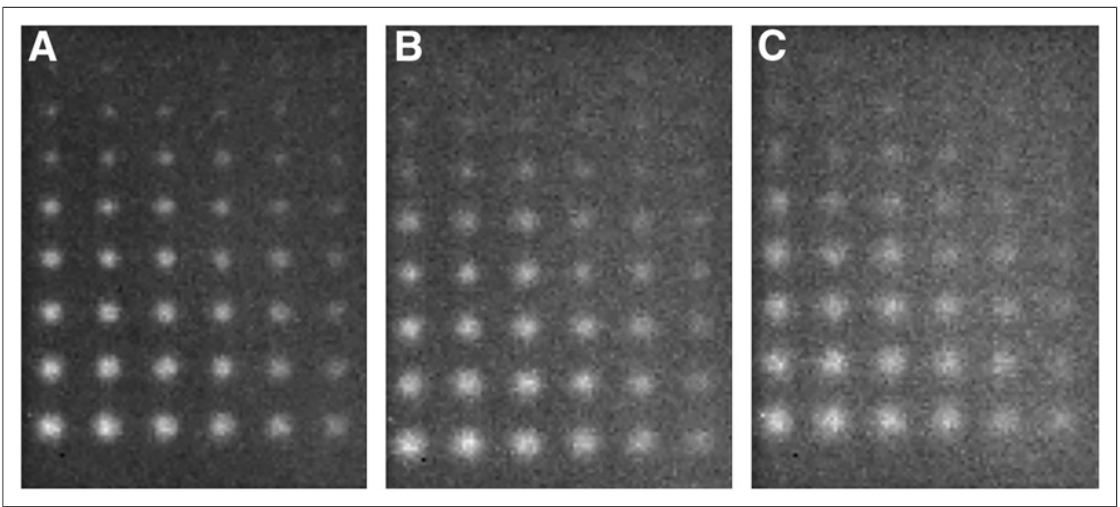

FIGURE 7. Sample contrast-detail phantom images. These images were acquired at distances of $1.5 \mathrm{~cm}(\mathrm{~A}), 3.0 \mathrm{~cm}(\mathrm{~B})$, and $4.5 \mathrm{~cm}(\mathrm{C})$ from collimator face. energy location is determined, the energy window is reduced to $2 \%$ or $2 \mathrm{keV}$ (depending on the available settings for the MBI system), and the peak count rate at this energy window is noted. Half the count rate is then determined, and the energy at which that rate occurs is located both below and above the peak location. The energy locations can be used to estimate the full width at half maximum and energy resolution of the system.

\section{Lesion Contrast Testing}

Measurement of the contrast at which lesions are detected by an MBI system is beneficial because the primary function of these systems is hot-spot detection. No ideal phantom for this purpose is commercially available, and there is currently no recommended phantom for MBI testing. For most MBI systems, a standard Jaszczak phantom (Data Spectrum Corp.) cannot be used because it is too large, although a mini-Jaszczak phantom might be used. We use an acrylic contrast-detail phantom (Merrimac Tool Co.) to evaluate the contrast-to-noise ratio (Fig. 6A). This phantom comprises a 3 -cm-thick central section and two 1.5-cm-thick spacer plates, for an overall thickness of $6 \mathrm{~cm}$ to match the typical thickness of the compressed breast in clinical MBI studies (2). The central section of the phantom is hollow and contains 48 cutout holes ranging from 3 to $10 \mathrm{~mm}$ in diameter. When a ${ }^{99 \mathrm{~m}} \mathrm{Tc}$ solution is added to this central section, it fills in the holes and background section of the phantom. The 2 spacer plates can be placed in front of or behind the central section to allow imaging of the holes at distances of 1.5, 3.0, and $4.5 \mathrm{~cm}$ from the collimator surface. Each hole diameter is represented 6 times, with hole depths of 6.0, 8.0, 9.9, 11.9, 13.8, and $15.8 \mathrm{~mm}$. The background region has a depth of $3.0 \mathrm{~mm}$. Hence, the phantom represents lesion-to-background ratios of 2.0:1, 2.7:1, 3.3:1, 4.0:1, 4.6:1, and 5.3:1.

For the lesion-contrast test, the central section of the phantom is filled with water and approximately $185 \mathrm{MBq}$ $(5 \mathrm{mCi})$ of $\mathrm{TcO}_{4}{ }^{-}$. The phantom is then shaken well, and the sides are tapped to dislodge air bubbles from the holes. For the first acquisition, the central section is placed directly on the face of the lower detector, and the 2 spacer plates are placed on top of the central section. If there is an upper detector, it is moved as closely as possible to the spacer plates so that the lower detector is $1.5 \mathrm{~cm}$ from the holes and the upper detector is $4.5 \mathrm{~cm}$ from the holes. The detectors are rotated slightly to move any remaining air bubbles out of the field of view (Fig. $6 \mathrm{~B})$, and 1 million counts are acquired. For the second acquisition, one of the spacer plates is placed beneath the central section so that both the lower detector and the upper detector are $3.0 \mathrm{~cm}$ from the holes, and 1 million 
TABLE 2

Suggested Number of Lesions Visualized in Contrast-Detail Phantom for Satisfactory, Marginal, and Fail Criteria

\begin{tabular}{llll}
\hline Distance & Satisfactory & Marginal & Fail \\
\hline $1.5 \mathrm{~cm}$ & 42 & 40 & $<40$ \\
$3.0 \mathrm{~cm}$ & 37 & 35 & $<35$ \\
$4.5 \mathrm{~cm}$ & 32 & 30 & $<30$ \\
\hline
\end{tabular}

counts are acquired. For the third acquisition, both spacer plates are placed beneath the central section so that the lower detector is $4.5 \mathrm{~cm}$ from the holes and the upper detector is $1.5 \mathrm{~cm}$ from the holes, and 1 million counts are acquired.

On completion of the 3 acquisitions (Fig. 7) for each detector (6 images total), the images are transferred to a viewing workstation. The number of lesions in each image is counted, and the results are compared with the criterion table (Table 2). It is recommended that this test be performed quarterly and that the results be compared with previous results to monitor system performance.

\section{DISCUSSION}

Modern MBI units differ from conventional $\gamma$-cameras in many ways. When performing physics testing, one needs to be aware of these differences. On the basis of our experience, we propose the following quality control tests for MBI systems: uniformity, spatial resolution, sensitivity, energy resolution, and lesion contrast. The frequency of testing should be as indicated by the accreditation body of the facility, with special consideration for any additional state regulations. As technology evolves, these quality control procedures may need to be redefined to accommodate improvements in MBI systems.

\section{CONCLUSION}

Routine physics testing of dedicated MBI equipment is important for verifying system specifications and monitoring changes in performance. As adoption of MBI grows, routine testing may be required for obtaining and maintaining accreditation from regulatory bodies.

\section{DISCLOSURE}

Michael O'Connor and Carrie Hruska have received royalties for licensed MBI technology per an agreement between Mayo Clinic and Gamma Medica. No other potential conflict of interest relevant to this article was reported.

\section{REFERENCES}

1. Hruska CB, O'Connor MK. Nuclear imaging of the breast: translating achievements in instrumentation into clinical use. Med Phys. 2013;40:050901.

2. Long Z, Conners AL, Hunt KN, Hruska CB, O'Connor MK. Performance characteristics of dedicated molecular breast imaging systems at low doses. Med Phys. 2016;43:3062-3070.

3. Siman W, Kappadath SC. Performance characteristics of a new pixelated portable gamma camera. Med Phys. 2012;39:3435-3444.

4. Weinmann AL, Hruska CB, O'Connor MK. Design of optimal collimation for dedicated molecular breast imaging systems. Med Phys. 2009;36:845-856.

5. NEMA Standards Publication NU 1-2012: Performance Measurements of Gamma Cameras. Rosslyn, VA: National Electrical Manufacturers Association; 2013.

6. Hruska CB, Weinmann AL, O'Connor MK. Proof of concept for low-dose molecular breast imaging with a dual-head CZT gamma camera. Part I. Evaluation in phantoms. Med Phys. 2012;39:3466-3475.

7. Hruska CB, Weinmann AL, Tello Skjerseth CM, et al. Proof of concept for lowdose molecular breast imaging with a dual-head CZT gamma camera. Part II. Evaluation in patients. Med Phys. 2012;39:3476-3483.

8. Sokole EB, Heckenberg A, Bergmann H. Influence of high-energy photons from cobalt-57 flood sources on scintillation camera uniformity images. Eur J Nucl Med. 1996;23:437-442.

9. Nuclear medicine accreditation program requirements. American College of Radiology website. https://www.acraccreditation.org/-/media/ACRAccreditation/ Documents/NucMed-PET/Nuclear-Medicine-Requirements.pdf?la=en. Revised February 27, 2018. Accessed June 28, 2018.

10. Bar phantoms. Biodex website. www.biodex.com/nuclear-medicine/products/ phantoms/bar-phantoms. Accessed June 28, 2018. 